\title{
Aktueller Algorithmus zur Diagnostik und Therapie des Brustimplantat-assoziierten anaplastischen großzelligen Lymphoms
}

\section{An algorithmic approach for diagnosis and treatment of breast implant-associated anaplastic large cell lymphoma}

Autoren

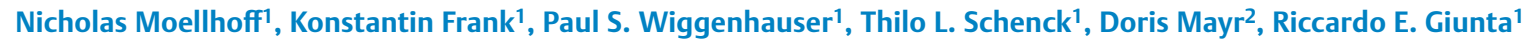

Institute

1 Abteilung für Hand-, Plastische und Ästhetische Chirurgie, Klinikum der Universität München, LMU München

2 Pathologisches Institut, Ludwig-Maximilians-Universität München, München Deutschland

\section{Schlüsselwörter}

Brustaugmentation, Ästhetisch-Plastische Chirurgie, Implantatregister, Brustimplantate, BIA-ALCL

Key words

Breast implant, breast augmentation, central registry, breast implant-associated anaplastic large cell lymphoma

eingereicht 9.12.2019

akzeptiert $\quad 21.01 .2020$

Bibliografie

DOI https://doi.org/10.1055/a-1111-8841

Handchir Mikrochir Plast Chir 2020; 52: 67-74

(c) Georg Thieme Verlag KG Stuttgart · New York

ISSN 0722-1819

Korrespondenzadresse

Nicholas Moellhoff

Abteilung für Hand-, Plastische und Ästhetische Chirurgie,

Klinikum der Universität München, LMU München

Pettenkoferstr. 8a, 80336 München

Email: Nicholas.Moellhoff@med.uni-muenchen.de

Tel: +49(0)89 4400-52697, Fax: +49(0)89 4400-54401

\section{ZUSAMMENFASSUNG}

Bei elektiven Operationen gilt es ein höchstes Maß an Sicherheit zu gewährleisten und Patienten ausführlich über die mit dem Eingriff verbundenen Risiken zu informieren. Insbesondere bei ästhetischen Eingriffen in der Plastischen Chirurgie ist es die Pflicht des Chirurgen eine ganzheitliche und umfassende
Aufklärung auch über mögliche Langzeitfolgen durchzuführen. In den letzten Jahren steht die Verwendung von Brustimplantaten zur ästhetischen Brustaugmentation oder zur Brustrekonstruktion nach Tumorresektion zunehmend im Fokus der Öffentlichkeit, nachdem sich weltweit die Fallzahlen von seltenen Brustimplantat-assoziierten Krankheitsbildern häufen und die Ursachen nicht vollständig geklärt sind. Das breast implant-associated anaplastic large cell lymphoma (engl. BIA-ALCL) wurde 2016 von der Weltgesundheitsorganisation als lymphatische Neoplasie klassifiziert.

Mittlerweile wurden weltweit knapp 800 Fälle, die vor allem texturierte Implantate betreffen, dokumentiert. Die maligne Erkrankung führt zu einer Verunsicherung von Patientinnen mit Implantaten. Daher ist es eine zentrale Aufgabe der Plastischen Chirurgen dieses seltene Krankheitsbild zu verstehen, es frühzeitig zu erkennen und klare Handlungsempfehlungen zu formulieren. Das vorliegende Manuskript soll den aktuellen Stand zum Vorgehen im Hinblick auf Diagnostik und Therapie des BIA-ALCL zusammenfassen.

\section{ABSTRACT}

Elective surgeries, especially aesthetic operations in plastic surgery, require surgeons to provide a holistic and comprehensive explanation of possible procedural risks and long-term consequences. Recently, the use of breast implants for aesthetic breast augmentation or breast reconstruction after tumour resection has gained public attention as the numbers of patients with rare breast implant-associated diseases have been rising worldwide, while the aetiopathogenesis remains unclear. BIA-ALCL was classified as a lymphatic neoplasm by the World Health Organization in 2016. Meanwhile, almost 800 cases have been documented, especially in patients that received textured implants. This malignant disease has led to a great level of uncertainty and scepticism in patients and the public. Hence, it is a central task of plastic surgeons to understand this rare disease, to recognise it at an early stage and help those affected by clear recommendations for action. The following manuscript summarises current approaches for diagnosis and treatment. 


\section{Einleitung}

Die Brustvergrößerung zählt zu den am häufigsten durchgeführten plastisch-chirurgischen Operationen weltweit. Jährlich werden etwa 1,5 Millionen Brustimplantate zur Brustvergrößerung eingesetzt [1], davon über 300000 in den USA [2] und 60000 in Deutschland [3]. Seit Einführung der Silikonimplantate haben mehrere Ereignisse, wie z. B. der PIP Skandal 2010, zu einer nachvollziehbaren Verunsicherung von Patientinnen, der Öffentlichkeit und Anwendern geführt. 2019 veröffentlichten Coroneos et al. die Langzeitergebnisse aus Nachuntersuchungen von 99993 Patienten, die mit Brustimplantaten versorgt wurden. Dazu wurden die Daten der U.S. Food and Drug Administration (FDA) post approval studies Datenbank ausgewertet, die zum Monitoring der Langzeitfolgen von Mentor und Allergan Implantaten initiiert wurde. Die Autoren stellten dabei ein fünf- bis achtfach höheres Auftreten von Autoimmunerkrankungen wie Rheumatoide Arthritis, Sklerodermie oder Sjögren Syndrom fest [4]. Zeitgleich mehren sich Fälle von der systemischen breast implant illness (BII) [5-7] oder vom Brustimplantat-assoziierten anaplastisch-großzelligen Lymphom (engl: breast implant-associated anaplastic large cell lymphoma; BIA-ALCL) [8-10].

Bei elektiven Operationen gilt es ein höchstes Maß an Sicherheit zu gewährleisten und Patienten ausführlich über die mit dem Eingriff verbundenen Risiken zu informieren. Insbesondere bei ästhetischen Eingriffen in der Plastischen Chirurgie ist es die Pflicht des Chirurgen eine ganzheitliche, umfassende und schonungslose Aufklärung auch über mögliche Langzeitfolgen durchzuführen. Behandelnde Plastische Chirurgen können sich dabei an den kürzlich durch die FDA angepassten Anforderungen bezüglich der Brustimplantat-Herstellerangaben orientieren. Hierin sollen fortan auch Warnhinweise zur Haltbarkeit der Brustimplantate, zum BIA-ALCL, zur Bll und auch zur chemischen Zusammenstellung der Implantate vorhanden sein $[11,12]$.

Das BIA-ALCL ist ein Lymphom, bei dem ein Zusammenhang zum Vorhandensein eines Brustimplantats nachgewiesen werden konnte und das histologisch immer in direkter Angrenzung zum Implantat auftritt $[13,14]$. Seit 2016 wird das BIA-ALCL von der Weltgesundheitsorganisation als lymphatische Neoplasie klassifiziert [15]. Da die Früherkennung der Erkrankung und das rasche Einleiten therapeutischer Maßnahmen wesentlich zur Überlebenszeit der Betroffenen beiträgt [10], ist das systematische und einheitliche Vorgehen zur Diagnostik und Therapie bei klinischem Verdacht entscheidend. Dazu ist eine interdisziplinäre Zusammenarbeit im Brustzentrum zwischen Plastischen Chirurgen, Gynäkologen, Haematoonkologen, Pathologen, Radiologen und Strahlentherapeuten notwendig. Noch sind die bisherigen wissenschaftlichen Erkenntnisse zum BIA-ALCL bedauerlicherweise selbst an vielen Brustzentren nicht vollständig bekannt. In diesem Manuskript soll die aktuelle wissenschaftliche Datenlage, sowie der an unserem Klinikum angewandte Algorithmus zur Diagnostik und Therapie zusammengefasst werden.

\section{Prävalenz und aktuelle Fallzahlen}

Nachdem erstmalig 1997 ein Fall von BIA-ALCL veröffentlicht wurde [16], konnten mittlerweile knapp 520 Fälle aus 25 Ländern in der PROFILE Datenbank der American Society of Plastic Surgeons
(ASPS) registriert werden [17]. Das Bundesinstitut für Arzneimittel und Medizinprodukte (BfArM) meldet im April 2019 weltweit ca. 800 Fälle bei etwa 35 Millionen Patientinnen mit Brustimplantaten [18]. Derzeit ist die Prävalenz, aufgrund der noch nicht erfolgten Umsetzung eines zentralen Implantatregisters in vielen Ländern [19] - so auch in Deutschland - nicht sicher abzuschätzen. Aus diesem Grund steht die Deutsche Gesellschaft der Plastischen, Rekonstruktiven und Ästhetischen Chirurgen (DGPRÄC) im engen Dialog mit dem Gesundheitsministerium und erarbeitet ein Konzept zur Etablierung eines einheitlichen und verpflichtenden zentralen Registers für Brustimplantate [19], zur Qualitätssicherung und Verbesserung der Patientensicherheit. Je nach Studienlage und Alter werden 1 bis 82 Fälle pro Millionen Brustimplantatträger beschrieben $[9,20]$, wobei von einer gewissen Dunkelziffer auszugehen ist und die epidemiologischen Daten sich noch in stetiger Veränderung befinden. So untersuchten beispielsweise Ghione et al. kürzlich in einer prospektiven klinischen Studie 3546 Patientinnen, die eine Brustrekonstruktion mit texturierten Implantaten erhielten und stellten in dieser Patientenpopulation eine Prävalenz von 1 Fall pro 443 Implantatträgerinnen fest [21].

Die folgenden Fallzahlen beruhen jeweils auf Informationen des BfArM. Im Juni 2019 betrug die Gesamtzahl der BIA-ALCL Fälle 741 weltweit. Davon waren 717 Fälle bei Patientinnen mit texturierten Implantaten und 24 Fälle bei Patientinnen mit glatten Implantaten aufgetreten, wobei aus dieser Zahl nicht hervorgeht, ob Patientinnen in der Vorgeschichte bereits texturierte Implantate hatten (siehe Abb. 1a). In Deutschland sind zum Zeitpunkt der Manuskripterstellung demnach 20 Fälle beschrieben, wie in $\mathbf{A b b}$. $1 \mathbf{b}$ gezeigt. In den Fällen mit bekannten Herstellerangaben waren $17 \mathrm{~Pa}$ tientinnen Implantatträger der Firma Allergan, zwei Patientinnen Implantatträger der Firma Polytech und eine Patientin Implantatträger der Firma Mentor ( $\triangleright$ Abb. 1c). Soweit bekannt waren alle genannten Implantate texturierte Implantate. Ein Fall von BIA-ALCL in Deutschland trat bei einer schwangeren Patientin mit laktierender Mamma auf [22]. Bis Juli 2019 liegen der FDA 33 Todesfälle durch BIA-ALCL weltweit vor [23]. Aktuell ist in Deutschland kein Todesfall beschrieben. Vor dem Hintergrund, dass in Deutschland ca. 60000 Implantataugmentationen jährlich durchgeführt werden [3], vorwiegend mit texturierten Implantaten, erscheinen die Fallzahlen hier recht gering. Dies ist am ehesten auf das sogenannte „under reporting“ durch aktuell noch fehlende zentrale Register zurückzuführen.

In einer weit überwiegenden Mehrzahl der Fälle sind texturierte Implantate mit BIA-ALCL assoziiert [23]. Im Gegensatz zu den in den USA in über $90 \%$ der Fälle verwendeten glattwandigen Implantaten, werden in Europa und Deutschland überwiegend Implantate mit texturierter Oberfläche genutzt [3, 24]. Nach einer Häufung von Erkrankungsfällen bei Patientinnen mit texturierten Implantaten der Firma Allergan, kam es im Dezember 2018 zu einem europaweiten Marktrückzug bestimmter texturierter Allergan Brustimplantate, welcher im Juli 2019 zu einem weltweiten Marktrückzug ausgeweitet wurde [18]. Ein expliziter Rückruf mit Austausch der Implantate erfolgte jedoch nicht. Derzeit sind entsprechend der Empfehlungen der DGPRÄC bei Allergan Implantat-Trägerinnen regelmäßige Kontrollen und nur bei klinischem Verdacht auf BIA-ALCL Diagnostik und Therapie einzuleiten [25]. Die DGPRÄC informiert Patientinnen seit vielen Jahren in einer speziellen Ru- 


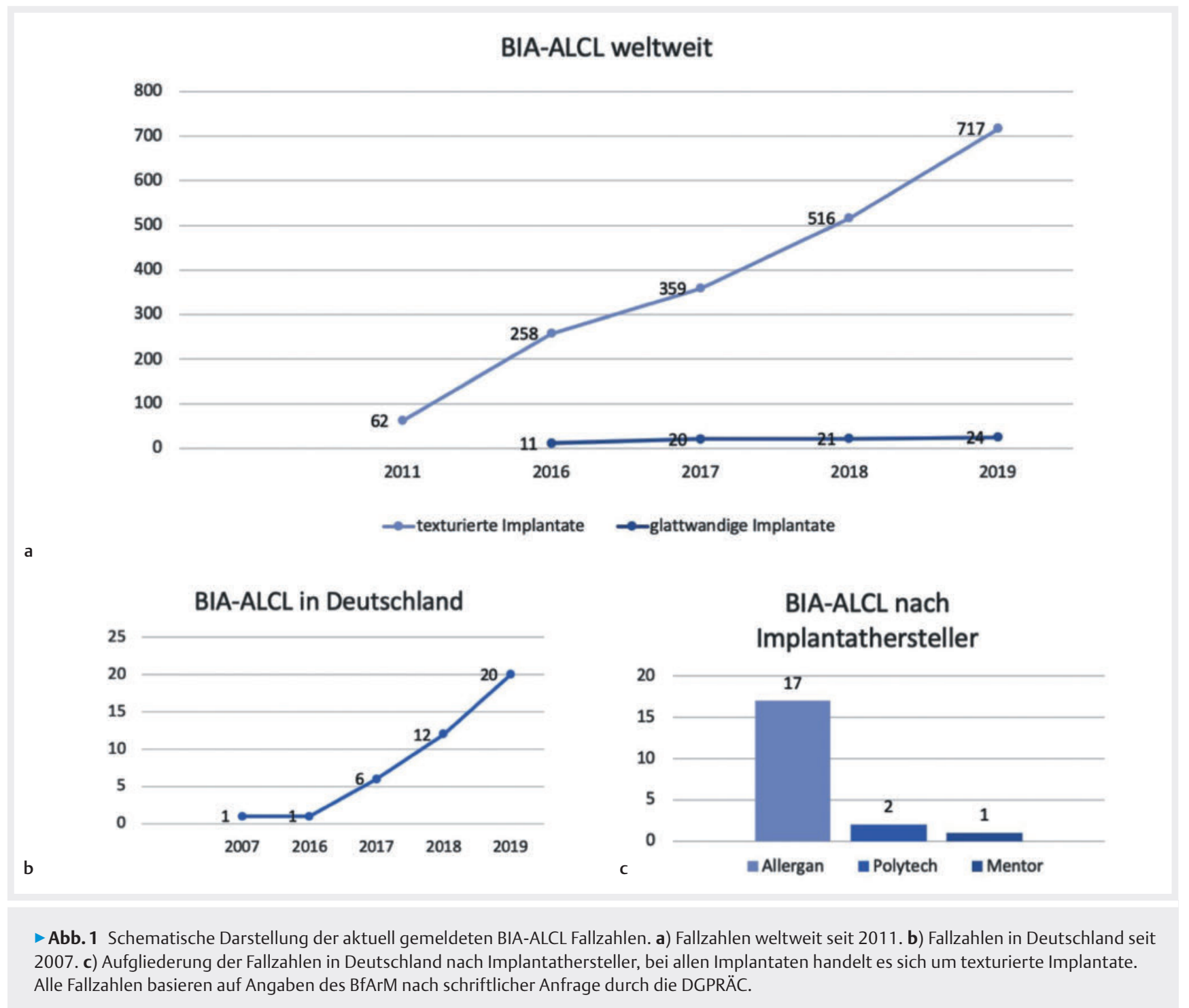

brik über das Krankheitsbild (https://www.dgpraec.de/patienten/ sonderthemen/alcl/).

\section{Pathogenese}

Das BIA-ALCL ist ein non-Hodgkin Lymphom der T-Zellreihe. Charakteristisch ist die monoklonale CD-30 positive anaplastische Zellpopulation, welche ALK (anaplastic lymphoma kinase) negativ ist und eine variable Expression T-Zell spezifischer Marker zeigt [26]. Die Pathogenese ist bis heute weitestgehend ungeklärt. Diskutiert wird, dass die Oberflächenbeschaffenheit und die chemische Zusammensetzung des Implantats eine chronische Inflammation induzieren, welche die Lymphomentstehung fördert [27-29]. Des Weiteren wird vermutet, dass ein bakterieller Biofilm die chronische Inflammation aufrechterhält. Die Oberflächenbeschaffenheit der Implantate steht dabei in direktem Bezug zur bakteriellen Besiedelung des Implantats. Studien konnten eine vermehrte bakterielle Besiedlung auf texturierten, im Vergleich zu glattwandigen Implantaten nachweisen [30, 31]. Aktueller Literatur zufolge, sind sogar alle bisherigen BIA-ALCL Fälle nur bei den Patientinnen auf- getreten, die zumindest einmal texturierte Implantate implantiert hatten [24,32-34]. Auch zwischen texturierten Implantaten unterscheidet sich die Ausprägung der Texturierung und somit die Rauheit der Oberfläche [30, 35], was als Erklärung für die unterschiedlichen herstellerspezifischen Fallzahlen dienen könnte. Mittlerweile sind auch zwei Fälle von ALCL nach Glutealaugmentation mit texturierten Silikonimplantaten beschrieben [36,37], sodass möglicherweise von einer implantatinduzierten Neoplasie auszugehen ist, die unabhängig von der Lokalisation des Implantats auftreten kann.

\section{Algorithmus zur Diagnostik}

Patientinnen stellen sich typischerweise 8-10 Jahre, aber mindestens 1 Jahr nach Brustimplantatversorgung mit meist ein- oder auch beidseitigem Serom und einhergehender Schwellung, Asymmetrie und Schmerzen vor, die nicht durch eine Infektion oder ein Trauma zu erklären sind. Seltener kann sich das BIA-ALCL auch als tastbare Tumorzellmasse oder axilläre Lymphadenopathie präsentieren [8, 34, 38]. Bei klinischem Verdacht sollte zunächst eine sonographische Untersuchung der Brust oder Bildgebung mittels MRT 
erfolgen: Klar zu unterscheiden sind Fälle mit einem Flüssigkeitssaum (> 5-10 ml) um das Implantat von solchen mit einer Tumormasse. Sonographisch wird auch nach vergrößerten Lymphknoten gesucht. Besteht sonographisch ein Flüssigkeitssaum um das Implantat erfolgt eine sonographisch kontrollierte Feinnadelaspirationszytologie (engl. Fine-needle aspiration cytology; FNAC) von bestenfalls zumindest $50 \mathrm{ml}$ pro Biopsie. Bei soliden Tumoren erfolgt je nach Tumorgröße eine Feinnadelbiopsie oder Exzisionsbiopsie. Anschließend werden die Präparate pathologisch aufbereitet. Zunächst werden Standardfärbung wie H\&E, Papanicolaou oder May-Grünwald-Giemsa im Ausstrichpräparat und Zytoblock angewandt und die Zellmorphologie beurteilt. Morphologisch zeichnen sich die neoplastischen Zellen beim BIA-ALCL durch mittelgroße bis große atypische Zellen mit reichlich eosinophilem Zytoplasma, unregelmäßigen Zellkernen mit prominenten Nukleoli aus. Die Zellen können exzentrische, nierenförmige Kerne aufweisen oder mehrkernig sein und Reed-Sternberg-Zellen ähneln [39, 40]. In der Regel machen die neoplastischen Zellen bis zu 70\% der Gesamtzellzahl der Seromflüssigkeit aus [26]. Anders als in vielen Publikationen beschrieben erfolgt bei Verdacht nicht automatisch die immunohistochemische Färbung von CD30, da dies kein spezifischer BIA-ALCL Marker ist und zu falsch-positiven Ergebnissen führen kann. Unser Vorgehen entspricht in dieser Hinsicht einer kürzlich publizierten histopathologischen Übersichtsarbeit zum BIA-ALCL von Jones et al. [26]. Nur wenn morphologisch auffällige und atypische Zellen erkannt werden, folgt zunächst die immunhistochemische Abklärung, zuerst mit CD30 und bei Bedarf mit CD68 oder CD163. Erst bei weiterbestehendem Verdacht sollten im Rahmen der Diagnosesicherung immunhistochemische Marker wie ALK, CD 2, CD 3 , CD 4, CD 5, CD 7, CD 8, CD 45 untersucht und letztlich eine T-Zell Klonalitätsanalyse durchgeführt werden. Sofern ein BIA-ALCL diagnostiziert wird, erfolgt durch den behandelnden Arzt die Meldung an das BfArM, den Hersteller, die FDA, das PROFILE Register der ASPS und die DGPRÄC.

Eine interdisziplinäre Zusammenarbeit im Brustzentrum zwischen Plastischen Chirurgen, Gynäkologen, Haematoonkologen, Pathologen, Radiologen und Strahlentherapeuten ist fortan immanent wichtig, um das weitere Vorgehen für die individuelle Patientin zu planen. Die Ausbreitungsdiagnostik wird mittels PET-CT durchgeführt [41], um beispielsweise eine Infiltration in Lymphknoten oder Thoraxwand zu erkennen. Ebenfalls erfolgen laborchemische Untersuchungen, die Bestimmung von Blutbild und Differenzialblutbild sowie ggf. eine Knochenmarkspunktion bei V. a. systemische ALCL, oder bei Patienten mit aggressivem Lokalbefund und Lymphknotenmetastasierung. - Abb. 2 fasst schematisch das relevante diagnostische und therapeutische Vorgehen zusammen.

Eine Stadieneinteilung erfolgt nach dem Tumorausmaß, der Lymphknoteninfiltration und Fernmetastasierung, entsprechend der erstmalig 2016 vom MD Anderson Cancer Center vorgeschlagenen TNM-Klassifizierung [10]. Entscheidend ist dabei vor allem die Infiltration der Kapsel um das Implantat und das Vorliegen von Zellaggregaten [42, 43]. - Abb. 3 zeigt die schematische TNM-Einteilung inklusive Staging. Wichtig ist, dass die Erkrankung bis T3 auf die Kapsel begrenzt ist und bei Infiltration über die Kapsel hinaus bereits ein T4 Tumor klassifiziert wird.

\section{Therapeutisches Vorgehen}

Die Therapie erfolgt bei uns ausgehend von den 2019 erschienenen NCCN Consensus Guidelines zum BIA-ALCL von Mark W. Clemens et al. [34]. Im Vordergrund steht die radikale chirurgische Therapie mit Implantatentfernung und vollständiger Kapsulektomie, sowie Exzision ggf. vorhandener Tumorzellmassen. Hierbei ist penibel darauf zu achten, die Kapsel nicht zu verletzen. Je nach Ausmaß des Tumors (in den Stadien T4), muss eine Ausweitung der Resektionsgrenzen erfolgen. Hierbei sollte das Vorgehen mit der Patientin und den vorhandenen operativen Ressourcen abgestimmt werden. Wenn aufgrund einer Infiltration der Thoraxwand, oder großer kutaner Anteile, eine ausgedehnte Resektion notwendig ist, wird die Anbindung an eine große plastisch-rekonstruktive Klinik dringend empfohlen. Eine Fadenmarkierung ist für die im Anschluss erfolgende histopathologische Untersuchung und mögliche Nachresektionen zwingend erforderlich. Kapsel und Implantat sollten der Pathologie unversehrt übergeben werden. Solide Tumoren sollten zusätzlich falls vorhanden entfernt und mit Orientierungsfäden versehen werden. Aktuell wird keine klare Aussage bezüglich Sicherheitsabständen oder der Empfehlung zur Sentinel-Lymphknotenbiopsie ausgesprochen [34]. Da in 2-4\% der Fälle ein bilaterales BIAALCL beschrieben ist, sollte mit der Patientin der prophylaktische Implantatwechsel inklusive Kapsulektomie auf der Gegenseite im gleichen Eingriff diskutiert werden [34].

Aus den Arbeiten von Clemens et al. (2016, 2019) geht hervor, dass die Rezidivrate nach vollständiger chirurgischer Resektion stadienabhängig in den Stadien T1-3 bei $0 \%$, im Stadium T4 jedoch bei 14,3\% liegt [10, 34]. Lokalrezidive treten dabei am häufigsten nach unvollständiger Resektion oder partieller Kapsulektomie auf.

Für fortgeschrittene Stadien und disseminierten Lymphknotenbefall fehlen klinische Studien im Hinblick auf die Wirksamkeit von adjuvanter Therapie. Diese richtet sich beim BIA-ALCL an den Therapieprotokollen von primär kutanem oder systemischem ALCL. Als adjuvante Therapie wird in fortgeschrittenen Stadien mit Lymphknotenbefall eine systemische Chemotherapie entsprechend der ECHELON II Studie mit Anthrazyklinen und Brentuximab Vedotin empfohlen [34]. Eine Radiotherapie mit 24 bis 36 Gray wird bei Lokalrezidiven, nicht-resektablem Tumor oder nicht-tumorfreien Resektionsrändern empfohlen [34]. Follow-Up Untersuchungen erfolgen alle 3 bis 6 Monate für die ersten 2 Jahre, danach entsprechend des klinischen Befunds. Eine Kontrastmittel-CT oder PET-CT Untersuchung sollte alle 6 Monate für 2 Jahre erfolgen [34].

Die durchschnittliche 5-Jahres-Überlebensrate beträgt unterschiedlichen Studie zufolge über $90 \%$ [10, 34, 44]. Das systemische ALK-negative ALCL, welches $2 \%$ bis $3 \%$ aller Non-Hodgkin-Lymphome ausmacht, hat im Gegensatz dazu eine wesentlich schlechtere Prognose mit einer 5-Jahres-Überlebensrate von etwa $20 \%$ bis $50 \%$ [45-47]. Das BIA-ALCL kann zwar disseminieren, bleibt jedoch in der Regel indolent, so dass die Unterscheidung vom de-novo-systemischem ALK-negativem ALCL wesentliche prognostische Implikationen hat.

Bislang fehlen gerichtliche Präzedenzfälle, die die Kostenfrage von Diagnostik und Behandlung bei Auftreten von BIA-ALCL nach ästhetischer Brustaugmentation als Selbstzahler abschließend klären. Wie bei allen ästhetischen Eingriffen gilt nach aktueller Gesetzeslage, dass wenn sich gemäß Paragraph 52 Absatz (2) Sozialgesetzbuch $\mathrm{V}$,[...] Versicherte eine Krankheit durch eine medizinisch 


\section{Algorithmus zur Diagnostik und}

Therapie

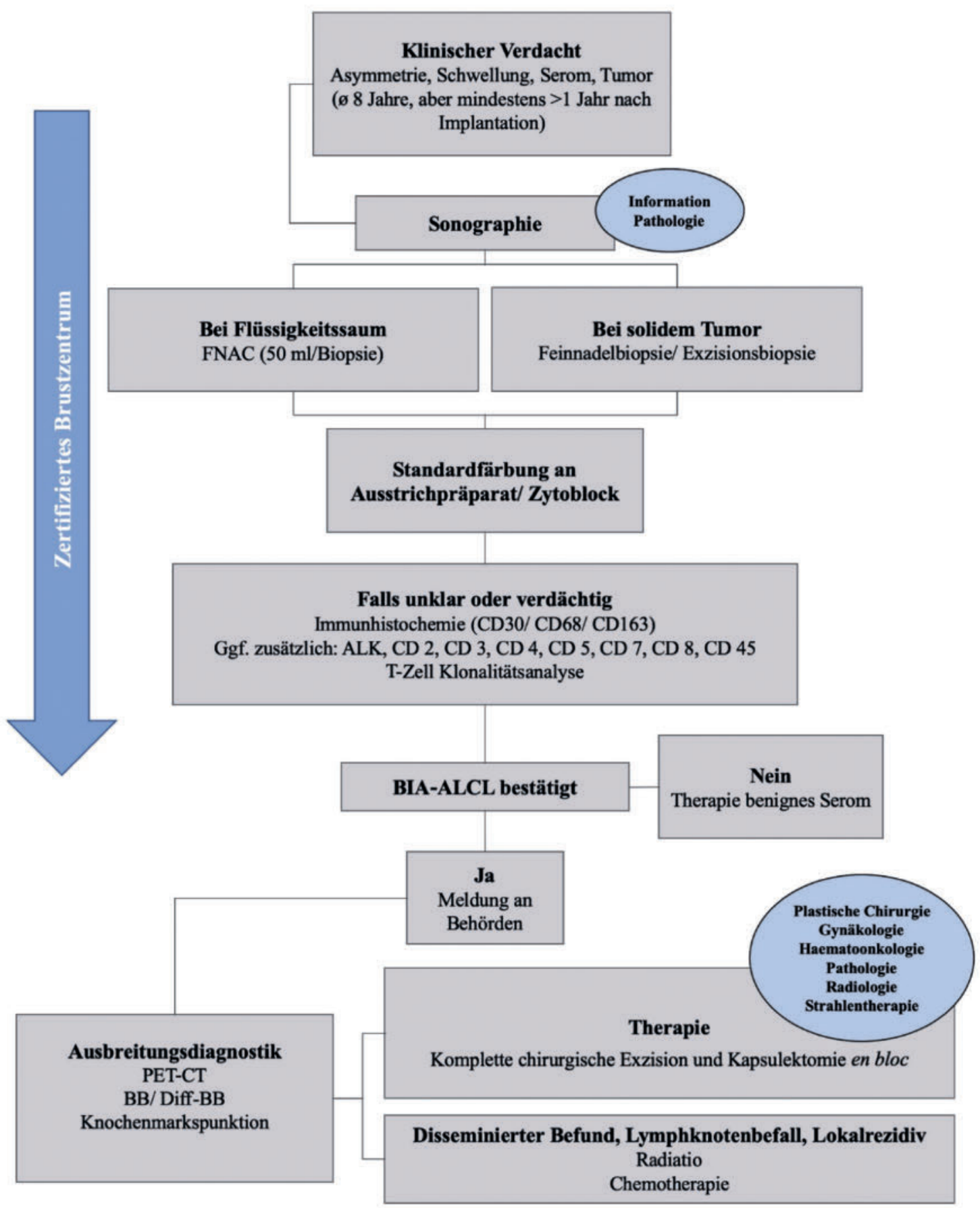

-Abb. 2 Schematische Vorgehensweise bezüglich Diagnostik und Therapie bei Verdacht auf BIA-ALCL. (basierend auf Daten aus [34,43])

nicht indizierte ästhetische Operation [...] zugezogen [haben], hat die Krankenkasse die Versicherten in angemessener Höhe an den Kosten zu beteiligen und das Krankengeld für die Dauer dieser Behandlung ganz oder teilweise zu versagen oder zurückzufordern“ [48].

\section{Fallbeispiel: Vorgehen bei klinischem Verdacht auf BIA-ALCL}

Eine 47-jährige Patientin stellte sich 10 Jahre nach ästhetischer Mammaugmentation alio loco mit texturierten Implantaten der Firma Allergan in unserer plastisch-chirurgischen Sprechstunde vor. Sie 


\begin{tabular}{|c|c|c|c|}
\hline \multicolumn{2}{|c|}{ TNM Tumorklassifikation } & \multicolumn{2}{|c|}{ Staging } \\
\hline \multicolumn{2}{|c|}{ Tumorausmaß (T) } & & \\
\hline $\mathrm{T} 1$ & Serom/ Zellen begrenzt auf der Luminalseite der Kapsel & IA & T1 N0 M0 \\
\hline $\mathrm{T} 2$ & Frühe Kapselinfiltration & IB & T2 N0 M0 \\
\hline T3 & Zellaggregate oder Zellschichten mit Kapselinfiltration & IC & T3 N0 M0 \\
\hline T4 & Lymphomausdehnung über die Kapsel hinaus & IIA & T4 N0 M0 \\
\hline \multicolumn{2}{|c|}{ Lymphknotenbefall (N) } & IIB & T1-3 N1 M0 \\
\hline No & Keine Lymphknoten befallen & III & T4 N1-2 M0 \\
\hline N1 & Ein regionaler Lymphknoten befallen & IV & $\mathrm{T}$ (jedes) $\mathrm{N}($ jedes $) \mathrm{M} 1$ \\
\hline $\mathrm{N} 2$ & Mehrere regionale Lymphknoten befallen & & \\
\hline \multicolumn{2}{|c|}{ Fernmetastasierung (M) } & & \\
\hline M0 & Keine Fernmetastasierung & & \\
\hline M1 & Metastasierung in Organe & & \\
\hline
\end{tabular}

- Abb.3 TNM Tumorklassifikation und Staging bei BIA-ALCL. (basierend auf Daten aus [34] und [10])
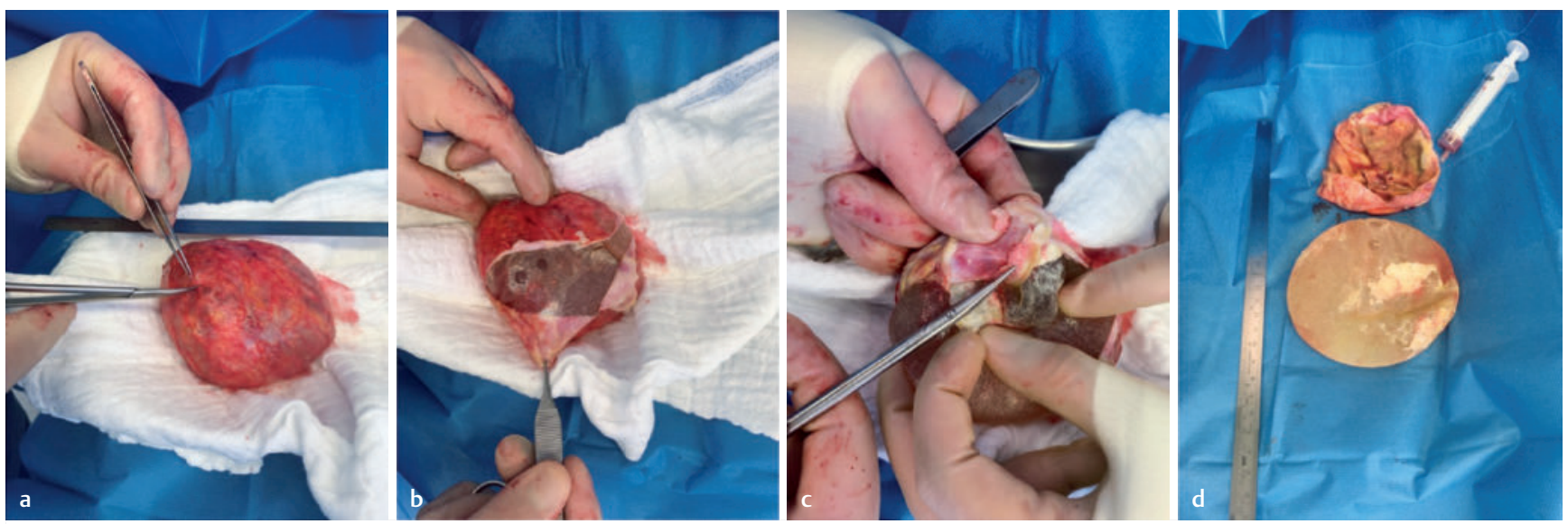

- Abb. 4 Fallbeispiel einer Patientin mit Verdacht auf BIA-ALCL. a) Befund nach en bloc Resektion von Implantat und Kapsel. b) Eröffnen der Kapsel und herauslösen des Implantats. c) Vereinzelte auffällige Zellnester im Bereich der Kapsel sichtbar. d) Komplette Entfernung der Kapsel vom Implantat vor Einsendung zur histopathologischen Untersuchung.

klagte über einseitige Schmerzen in der rechten Brust. In der klinischen Untersuchung fiel eine Kapselfibrose im Baker Stadium III auf. Die Brust tastete sich verhärtet und eine beginnende Verformung war sichtbar. Sonographisch zeigte sich ein Flüssigkeitssaum um das Brustimplantat. In der FNAC konnten 50 ml Serom zur histopathologischen Untersuchung gewonnen werden. Die Kollegen der Pathologie wurden bezüglich des Verdachts auf BIA-ALCL informiert. In der histopathologischen Untersuchung konnte der Verdacht auf BIAALCL jedoch aufgrund der Zellmorphologie nicht bestätigt werden. In dem Serom wurden Lymphozyten, Makrophagen und polymorphe neutrophile Granulozyten dargestellt, sodass am ehesten von einem reaktiven Serom, ohne atypische Zellen, im Rahmen der Kapselfibrose auszugehen war. Aufgrund des schmerzhaften Lokalbefunds empfahlen wir der Patientin dennoch einen Implantatwechsel mit Kapsulektomie. - Abb. 4 zeigt das vollständig und in toto entfernte Brustimplantat inklusive der umhüllenden Kapsel. Das Implantat lässt sich vollständig aus der Kapsel schälen, intrakapsulär zeigen sich dabei Ansammlungen von verdächtigen Zellnestern ( $\triangleright$ Abb.4c). Die histopathologische Untersuchung und immunhistochemische Färbung (CD30, ALK) konnte jedoch ein BIA-ALCL endgültig ausschließen. 


\section{Schlussfolgerung}

Bei Verdacht auf das Vorliegen eines BIA-ALCL gilt es strukturiert und organisiert vorzugehen. Der in diesem Manuskript beschriebene Algorithmus zur Diagnostik und Therapie fasst unsere Vorgehensweise entsprechend der aktuellen Literaturlage zusammen. Wichtig ist, dass alle relevanten Fachabteilungen frühzeitig informiert und involviert werden und ein interdisziplinäres Diagnoseund Behandlungskonzept vorliegt. Die plastisch-chirurgische totale Resektion von Kapsel, Implantat und ggf. vorhandenen Tumorzellmassen steht bei der Therapie im Vordergrund. Zusammenfassend bleibt festzuhalten, dass das BIA-ALCL zwar weiterhin als sehr seltene Erkrankung anzusehen ist, aber auf Grund der Verantwortung für die Patientensicherheit ein kompetentes Aufklärungs-, Diagnose und Behandlungskonzept vorliegen sollte.

\section{Interessenkonflikt}

Die Autoren geben an, dass kein Interessenkonflikt besteht.

\section{Autorinnen/Autoren}

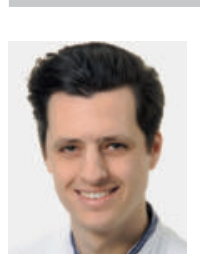

\section{Nicholas Moellhoff}

geb. 1991 in Limassol, Zypern; 2010-2017 Studium der Humanmedizin an der Ludwig-Maximilians-Universität München; 2017 Approbation als Arzt. Seit 2017 Assistenzarzt der Abteilung für Hand-, Plastische- und Ästhetische Chirurgie am Klinikum der Universität München (LMU), Leiter: Univ-Prof. Dr. med. Riccardo Giunta.

\section{Literatur}

[1] https://www.isaps.org/wp-content/uploads/2018/10/ISAP2016_17_ comparison.pdf; Zugegriffen: 02.12.2019.

[2] The American Society for Aesthetic Plastic Surgery's Cosmetic Surgery National Data Bank: Statistics 2018. Aesthet Surg J 2019; 39: 1-27

[3] https://www.dgpraec.de/wp-content/uploads/2019/04/2019-03-28Statement_ALCL.pdf; Zugegriffen: 02.12.2019

[4] Coroneos C], Selber JC, Offodile AC, 2nd et al. US FDA Breast Implant Postapproval Studies: Long-term Outcomes in 99993 Patients. Ann Surg 2019; 269: 30-36

[5] Magnusson MR, Cooter RD, Rakhorst $\mathrm{H}$ et al. Breast Implant Illness: A Way Forward. Plast Reconstr Surg 2019; 143: 74S-81S

[6] Rohrich RJ, Kaplan J, Dayan E. Silicone Implant Illness: Science versus Myth? Plast Reconstr Surg 2019; 144: 98-109

[7] Tang SY, Israel JS, Afifi AM. Breast Implant Illness: Symptoms, Patient Concerns, and the Power of Social Media. Plast Reconstr Surg 2017, DOI: $10.1097 / P R S .0000000000003785$

[8] Loch-Wilkinson A, Beath KJ, Knight RJW et al. Breast Implant-Associated Anaplastic Large Cell Lymphoma in Australia and New Zealand: High-Surface-Area Textured Implants Are Associated with Increased Risk. Plast Reconstr Surg 2017; 140: 645-654

[9] de Boer M, van Leeuwen FE, Hauptmann M et al. Breast Implants and the Risk of Anaplastic Large-Cell Lymphoma in the Breast. JAMA Oncol 2018; 4: 335-341
[10] Clemens MW, Medeiros LJ, Butler CE et al. Complete Surgical Excision Is Essential for the Management of Patients With Breast Implant-Associated Anaplastic Large-Cell Lymphoma. Journal of clinical oncology: official journal of the American Society of Clinical Oncology 2016; 34: 160-168

[11] https://www.fda.gov/regulatory-information/search-fda-guidancedocuments/breast-implants-certain-labeling-recommendations-improve-patient-communication; Zugegriffen 02.12.2019

[12] Clemens MW, Miranda RN, Butler CE. Breast Implant Informed Consent Should Include the Risk of Anaplastic Large Cell Lymphoma. Plast Reconstr Surg 2016; 137: 1117-1122

[13] Ebner PJ, Liu A, Gould DJ et al. Breast implant-associated anaplastic large cell lymphoma, a systematic review and in-depth evaluation of the current understanding. J Surg Oncol 2019; 120: 573-577

[14] Roden AC, Macon WR, Keeney GL et al. Seroma-associated primary anaplastic large-cell lymphoma adjacent to breast implants: an indolent T-cell lymphoproliferative disorder. Modern pathology : an official journal of the United States and Canadian Academy of Pathology, Inc 2008; $21: 455-463$

[15] Swerdlow SH, Campo E, Pileri SA et al. The 2016 revision of the World Health Organization classification of lymphoid neoplasms. Blood 2016; 127: $2375-2390$

[16] Keech JA, Jr., Creech BJ. Anaplastic T-cell lymphoma in proximity to a saline-filled breast implant. Plast Reconstr Surg 1997; 100: 554-555

[17] McCarthy CM, Loyo-Berrios N, Qureshi AA et al. Patient Registry and Outcomes for Breast Implants and Anaplastic Large Cell Lymphoma Etiology and Epidemiology (PROFILE): Initial Report of Findings, 2012-2018. Plast Reconstr Surg 2019; 143: 65S-73S

[18] https://www.bfarm.de/SharedDocs/Risikoinformationen/Medizinprodukte/DE/Brustimplantate_ALCL_FDA.html; Zugegriffen: 24.11.2019

[19] Prantl L, von Fritschen U, Liebau ] et al. [Concept for a National Implant Registry to Improve Patient Safety]. Handchir Mikrochir Plast Chir 2016; 48: 320-329

[20] de Jong D, Vasmel WL, de Boer JP et al. Anaplastic large-cell lymphoma in women with breast implants. Jama 2008; 300: 2030-2035

[21] Ghione P, Cordeiro PG, Ni A et al. Risk of breast implant-associated anaplastic large cell lymphoma (BIA-ALCL) in a cohort of 3546 women prospectively followed after receiving textured breast implants. Journal of Clinical Oncology 2019; 37: 1565-1565

[22] Messingschlager KJ, Mamarvar M. [Breast-implant-associated anaplastic large cell lymphoma (BIA-ALCL) in a lactating breast]. Handchir Mikrochir Plast Chir 2019; 51: 151-152

[23] https://www.fda.gov/medical-devices/breast-implants/medical-device-reports-breast-implant-associated-anaplastic-large-cell-lymphoma; Zugegriffen: 02.12.2019

[24] Swanson E. Plastic Surgeons Defend Textured Breast Implants at 2019U. S. Food and Drug Administration Hearing: Why It Is Time to Reconsider. Plast Reconstr Surg Glob Open 2019; 7: e2410

[25] https://www.dgpraec.de/wp-content/uploads/2018/12/2018-12-20Patienteninformation_ALLERGAN.pdf; Zugegriffen: 02.12.2019

[26] Jones JL, Hanby AM, Wells $C$ et al. Breast implant-associated anaplastic large cell lymphoma (BIA-ALCL): an overview of presentation and pathogenesis and guidelines for pathological diagnosis and management. Histopathology 2019, DOI: 10.1111/his.13932

[27] Bizjak M, Selmi C, Praprotnik S et al. Silicone implants and lymphoma: The role of inflammation. J Autoimmun 2015; 65: 64-73

[28] Hu H, Johani K, Almatroudi A et al. Bacterial Biofilm Infection Detected in Breast Implant-Associated Anaplastic Large-Cell Lymphoma. Plast Reconstr Surg 2016; 137: 1659-1669

[29] George EV, Pharm J, Houston C et al. Breast implant-associated ALKnegative anaplastic large cell lymphoma: a case report and discussion of possible pathogenesis. Int J Clin Exp Pathol 2013; 6: 1631-1642 
[30] Mempin M, Hu H, Chowdhury D et al. The A, B and C's of Silicone Breast Implants: Anaplastic Large Cell Lymphoma, Biofilm and Capsular Contracture. Materials (Basel) 2018; 11

[31] Jones P, Mempin M, Hu H et al. The Functional Influence of Breast Implant Outer Shell Morphology on Bacterial Attachment and Growth. Plast Reconstr Surg 2018; 142: 837-849

[32] Doren EL, Miranda RN, Selber JC et al. U. S. Epidemiology of Breast Implant-Associated Anaplastic Large Cell Lymphoma. Plast Reconstr Surg 2017; 139: 1042-1050

[33] Brody GS. Anaplastic Large Cell Lymphoma Occurring in Women with Breast Implants: Analysis of 173 Cases. Plast Reconstr Surg 2015; 136 : 553e-554e

[34] Clemens MW, Jacobsen ED, Horwitz SM. 2019 NCCN Consensus Guidelines on the Diagnosis and Treatment of Breast Implant-Associated Anaplastic Large Cell Lymphoma (BIA-ALCL). Aesthet Surg J 2019; 39: S3-S13

[35] James GA, Boegli L, Hancock J et al. Bacterial Adhesion and Biofilm Formation on Textured Breast Implant Shell Materials. Aesthetic Plast Surg 2019; 43: 490-497

[36] Shauly O, Gould D], Siddiqi I et al. The First Reported Case of Gluteal Implant-Associated Anaplastic Large Cell Lymphoma (ALCL). Aesthet Surg J 2019; 39: NP253-NP258

[37] Mendes ], Mendes Maykeh VA, Frascino LF et al. Gluteal Implant Associated Anaplastic Large Cells Lymphoma. Plast Reconstr Surg 2019, DOI: 10.1097/PRS.0000000000005910

[38] Brody GS, Deapen D, Taylor CR et al. Anaplastic large cell lymphoma occurring in women with breast implants: analysis of 173 cases. Plast Reconstr Surg 2015; 135: 695-705

[39] Laurent C, Delas A, Gaulard P et al. Breast implant-associated anaplastic large cell lymphoma: two distinct clinicopathological variants with different outcomes. Annals of oncology : official journal of the European Society for Medical Oncology/ESMO 2016; 27: 306-314

[40] Di Napoli A, Pepe G, Giarnieri E et al. Cytological diagnostic features of late breast implant seromas: From reactive to anaplastic large cell lymphoma. PloS one 2017; 12: e0181097

[41] Berlin E, Singh K, Mills C et al. Breast Implant-Associated Anaplastic Large Cell Lymphoma: Case Report and Review of the Literature. Case Rep Hematol 2018; 2018: 2414278

[42] Clemens MW, Brody GS, Mahabir RC et al. How to Diagnose and Treat Breast Implant-Associated Anaplastic Large Cell Lymphoma. Plast Reconstr Surg 2018; 141: 586e-599e

[43] Kricheldorff J, Fallenberg EM, Solbach C et al. Breast Implant-Associated Lymphoma. Dtsch Arztebl Int 2018; 115: 628-635

[44] Miranda RN, Aladily TN, Prince HM et al. Breast implant-associated anaplastic large-cell lymphoma: long-term follow-up of 60 patients. Journal of clinical oncology : official journal of the American Society of Clinical Oncology 2014; 32: 114-120

[45] Zimmerman A, Locke FL, Emole J et al. Recurrent Systemic Anaplastic Lymphoma Kinase-Negative Anaplastic Large Cell Lymphoma Presenting as a Breast Implant-Associated Lesion. Cancer Control 2015; 22: 369-373

[46] Vose J, Armitage J, Weisenburger D et al. International peripheral T-cell and natural killer/T-cell lymphoma study: pathology findings and clinical outcomes. Journal of clinical oncology : official journal of the American Society of Clinical Oncology 2008; 26: 4124-4130

[47] Kao EY, Lynch DT. Cancer, Lymphoma, ALK Negative Anaplastic Large Cell. In StatPearls. Treasure Island (FL); 2019

[48] https://dejure.org/gesetze/SGB_V/52.html; Zugegriffen: 21.12.2019 